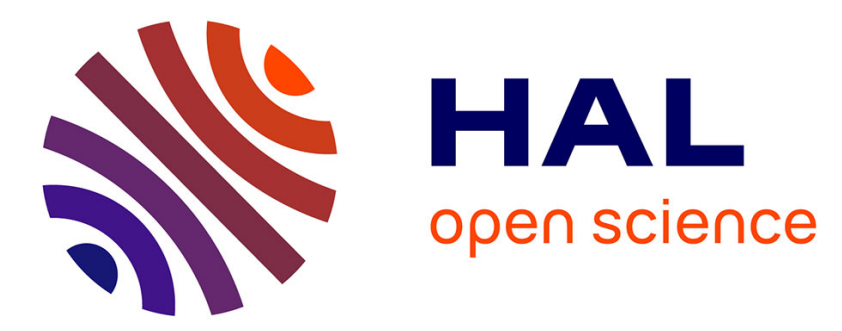

\title{
L'hydrodynamique, une composante importante dans la croissance des cristaux en solution
}

\author{
F. Lefaucheux, M. C. Robert
}

\section{To cite this version:}

F. Lefaucheux, M. C. Robert. L'hydrodynamique, une composante importante dans la croissance des cristaux en solution. Revue de Physique Appliquée, 1979, 14 (12), pp.949-959. 10.1051/rphysap:019790014012094900 . jpa-00244685

\section{HAL Id: jpa-00244685 https://hal.science/jpa-00244685}

Submitted on 1 Jan 1979

HAL is a multi-disciplinary open access archive for the deposit and dissemination of scientific research documents, whether they are published or not. The documents may come from teaching and research institutions in France or abroad, or from public or private research centers.
L'archive ouverte pluridisciplinaire HAL, est destinée au dépôt et à la diffusion de documents scientifiques de niveau recherche, publiés ou non, émanant des établissements d'enseignement et de recherche français ou étrangers, des laboratoires publics ou privés. 


\title{
REVUE DE PHYSIQUE APPLIQUÉE
}

Classification

Physics Abstracts

$61.70 \mathrm{~J}-61.70 \mathrm{Y}-62.10-47.15$

\section{L'hydrodynamique, une composante importante (*) dans la croissance des cristaux en solution}

\author{
F. Lefaucheux et M. $\dot{C}$. Robert \\ Laboratoire de Minéralogie Cristallographie, associé au C.N.R.S., Université P.-et-M.-Curie, Paris, France
}

(Reçu le 2 juillet, accepté le 24 septembre 1979)

\begin{abstract}
Résumé. - Différents aspects de la croissance des cristaux en solution sont successivement passés en revue pour montrer le rôle de l'environnement hydrodynamique.
\end{abstract}

Abstract. - Different aspects of the solution growth of crystals are reviewed in order to examine the role of the hydrodynamic environment.

Introduction. - Les techniques de croissance en solution utilisent généralement divers systèmes d'agitation de la solution. Dans une solution non agitée, le transfert de matière de la solution vers le cristal se fait principalement par diffusion. Mais Goldsztaub et Itti [1] ont montré, par des méthodes interférométriques, que la convection naturelle participe également au transport de matière vers le cristal, de telle sorte qu'il n'est pas possible de réaliser sur terre, dans un récipient où les convections ne sont pas bloquées, une croissance par diffusion pure. Il est possible d'augmenter les courants de convection en forçant la solution vers le cristal, ou en agitant le cristal dans la solution; l'agitation joue alors un double rôle : homogénéisation de la solution en concentration et température et renouvellement régulier de celle-ci au niveau du cristal.

Les dispositifs expérimentaux d'agitation sont extrêmement nombreux. Petrov et al. [2] présentent une revue assez complète des divers systèmes d'agitation et de vibration du cristal dans la solution. Des techniques de rotation alternée sans accélération brutale peuvent conduire à de bons résultats $[3,38]$. Dans le but de comprendre le rôle de l'agitation, des études systématiques de l'influence d'un flux de solution sur la croissance ont été entreprises.

Elles sont essentiellement de deux types :

- Des études poursuivant un but plutôt théorique, à savoir élucider le mécanisme de croissance; il

$\left(^{*}\right)$ Exposé présenté à la réunion annuelle du Groupe Français de Croissance Cristalline (GFCC) à Bordeaux, 25-27 octobre 1978. faut alors opérer dans des conditions propres : température et sursaturation constantes, flux de solution bien défini, donc opérer sur des cristaux de petite taille sur lesquels on dirige un flux de solution sursaturée. A ce type appartiennent la plupart des études de cinétique de croissance et de nucléation.

- Des études qui se sont développées à partir d'un problème pratique de croissance de monocristaux. Le but poursuivi est de déterminer quelles sont les conditions à fixer pour obtenir le meilleur cristal. Il s'agit donc d'études de qualité cristalline en fonction de l'agitation. Le cristal est agité dans la solution, les conditions de croissance ne sont, en général, pas stationnaires : la température varie au cours du temps, la sursaturation change le long des faces qui sont de grande taille et on ne définit qu'un flux moyen de solution arrivant sur une face. Voyons d'abord des études du premier type.

I. Vitesse de croissance. - La plupart des études de cinétique de croissance mentionnent une augmentation de la vitesse de croissance avec le degré d'agitation. Citons les études de Byteva sur l'ADP [4], de Sip et Vanicek [5] sur l'EDP, DPT, de Bennema [6], Denk et Botsaris [7, 8] et Mullin et Garside [9] sur l'alun de potassium.

Le dispositif employé par ces derniers est une cellule chauffante dans laquelle on place un germe de 1 à $2 \mathrm{~mm}$, recevant normalement à la face étudiée un flux de solution de vitesse $u$. Le domaine de sursaturation $(\sigma=\Delta c / c)$ examiné va de $1 \%$ à $20 \%$, à $T=32^{\circ} \mathrm{C}$ pour des flux variant de $3 \times 10^{-3}$ à $150 \times 10^{-3} \mathrm{~m} / \mathrm{s}$. La vitesse de croissance des faces 


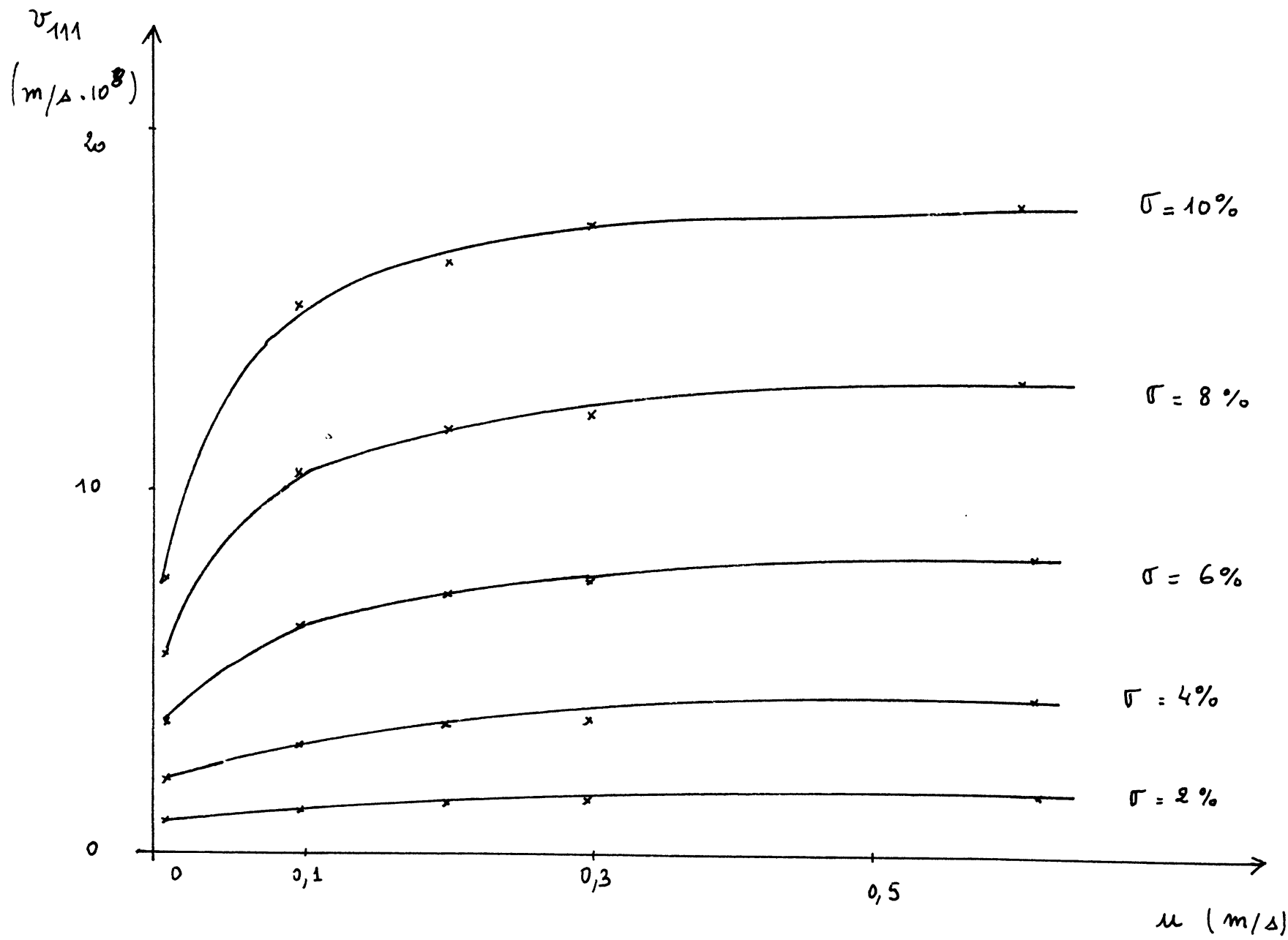

a)

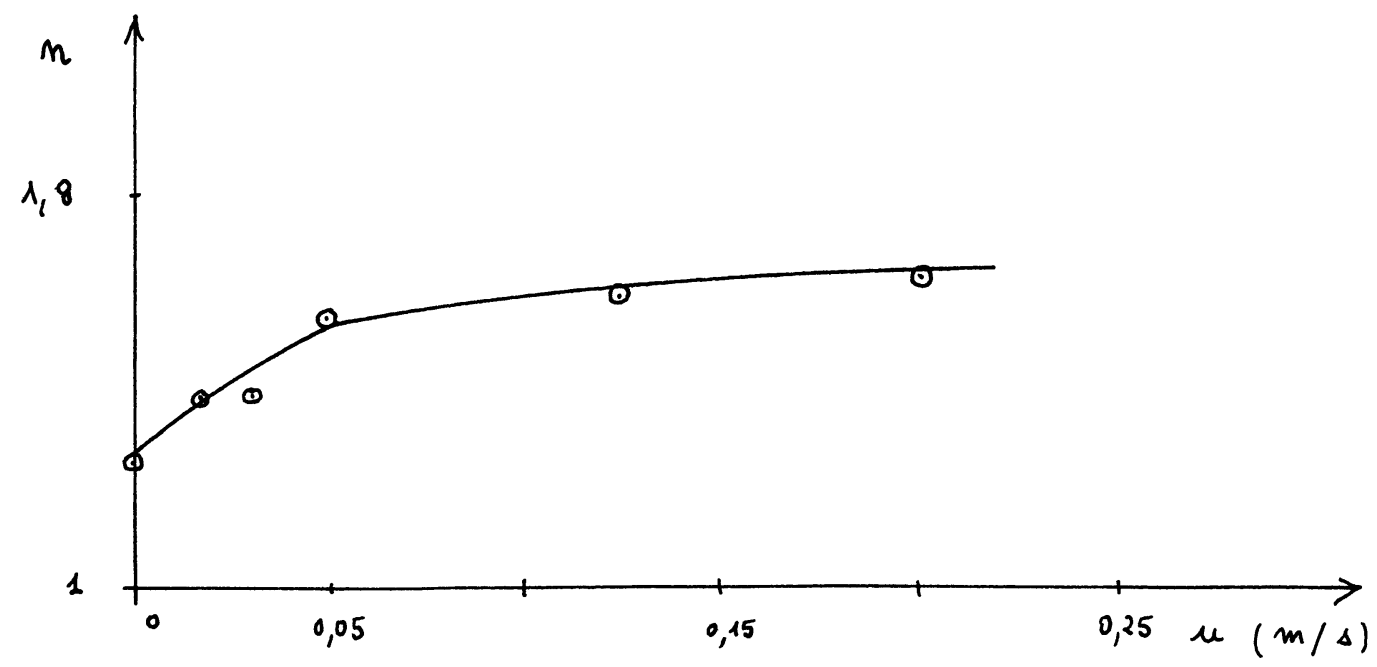

b)

Fig. 1. - a) Effet de la vitesse de la solution $u$ sur la croissance des faces (111) de l'alun de potassium [10]. $b$ ) Variation de $n$ avec la vitesse de la solution [9].

[a) The effect of solution velocity $u$ on the growth rate of (111) faces of potash alum [10]. b) Variation of $n$ with solution velocity [9].] 
(111) augmente, pour une sursaturation donnée, avec $u$ jusqu'à une limite (Fig. $1 a$ ) au-delà de laquelle l'agitation n'a plus d'effet. Ces auteurs trouvent une loi de la forme $v_{111}=K \sigma^{n}$ où $n$ varie avec le flux (Fig. 1b), ceci implique une modification du processus de croissance.

Ces résultats ont été interprétés et comparés [10] aux données de la littérature sur ce même cristal. On peut décomposer le processus de croissance en solution principalement en deux étapes, la plus lente imposant sa vitesse à l'ensemble. La première étape consiste en une diffusion de la solution vers le cristal à travers une couche limite d'épaisseur $\delta$, puis ont lieu à la surface divers processus d'intégration [11]. Car $\delta$ diminue avec $u$ comme l'ont montré divers auteurs $[12,13,14]$, suivant une formule du type :

$$
\delta=\left[K\left(\frac{\eta}{\rho_{\mathrm{s}} D}\right)^{1 / 3}\left(\frac{\rho_{\mathrm{s}} u}{\eta l}\right)^{1 / 2}\right]^{-1}
$$

$f=$ longueur de la face recevant le flux,

$\eta=$ viscosité de la solution,

$D=$ coefficient de diffusion du soluté,

$\rho_{\mathrm{s}}=$ densité de la solution.

Pour de faibles vitesses de flux, c'est la diffusion à travers la couche limite qui impose sa vitesse; sous l'effet d'une augmentation du flux, $\delta$ diminue, la vitesse de croissance augmente. Mais à faible sursaturation et agitation forte, c'est l'étape d'intégration en surface qui contrôle la vitesse, d'où l'existence du palier observé sous forte vitesse de flux.

Les études de cinétique de croissance sont difficiles et presque tous les auteurs signalent d'importantes dispersions diversement interprétées. Punin et Petrov [15], en mesurant des vitesses de croissance de $\mathrm{KCl}$ à diverses températures, décèlent des anomalies qu'ils relient à des variations de structure de la solution. Belyustin et Portnov [16] utilisent des sphères monocristallines d'alun de potassium pour mesurer des vitesses absolues de croissance en fonction de la vitesse de rotation du cristal dans la solution. Ils montrent notamment que la vitesse des faces octaédriques augmente bien avec le flux de solution mais dépend aussi de la nature des faces environnantes; ils interprètent leurs résultats par l'existence d'une couche limite dont la concentration varie d'une face à l'autre. Petrov [17] note que lors de la croissance du nitrate de potassium, des faces homologues poussent à des vitesses très différentes alors que les conditions extérieures sont apparemment les mêmes, une augmentation de la vitesse de croissance semblant liée à la présence d'inclusions. Alors que les résultats concernant les faces octaédriques de l'alun de potassium sont cohérents, Mullin et Garside [9] trouvent des dispersions importantes pour les faces du cube ; ils attribuent ces écarts à une phase initiale rapide due à une face imparfaite contenant de nombreux sites de croissance. Byteva [4], Shabalin et
Inyushkin [18] montrent sur $\mathrm{ADP}, \mathrm{NaNO}_{3}$, $\mathrm{K}_{4} \mathrm{Fe}(\mathrm{CN})_{6}$, qu'une augmentation de la vitesse d'agitation au-delà d'une certaine limite (beaucoup plus grande que celle utilisée dans les expériences précédentes) conduit à une diminution de la vitesse de croissance d'autant plus importante que la structure cristalline est plus complexe : ils supposent que les unités de croissance formées dans la couche limite sont emmenées loin du cristal quand la vitesse d'agitation est élevée, d'où une diminution de la vitesse de croissance.

De plus, pour les vitesses d'agitation utilisées, le flux peut ne pas être laminaire mais turbulent. Rodriguez-Clemente [19] a déterminé sur $\mathrm{NaCl}$ pour quelles vitesses de flux celui-ci devient turbulent. Il soumet un cristal germe à un flux orienté de telle sorte qu'une face $\{100\}$ reçoit un flux normal donc turbulent alors qu'une face homologue reçoit un flux tangentiel. En comparant les cinétiques de croissance, il montre qu'au-delà d'une certaine vitesse du flux, il n'y a plus de différence entre les deux faces. Pour caractériser le flux il utilise un nombre de Reynolds calculé pour le cristal. Au-delà d'une valeur critique de ce nombre de Reynolds, l'anisotropie dans l'apport de matière est compensée par la turbulence de la solution au voisinage du cristal.

$\mathrm{Au}$ total, les anomalies observées sont le plus souvent attribuées à des différences de qualité cristalline sans toutefois pouvoir préciser puisqu'il s'agit de microcristaux. Certains auteurs signalent des anomalies structurales de la solution.

L'agitation de la solution peut avoir aussi une forte influence sur la nucléation.

II. Nucléation. - Depuis longtemps déjà, de nombreux auteurs (cf. par ex. [20, 21]) ont montré que le nombre des cristaux augmente beaucoup quand la solution est agitée, ceci en présence d'un cristal germe ou d'un modèle amorphe. Il s'agit donc de préciser comment l'agitation modifie la nucléation. La nucléation est un phénomène complexe : on distingue la nucléation homogène de la nucléation hétérogène. La première conduit à la formation, au sein de la solution, sans particule externe, de noyaux quand l'agglomération des molécules (ou des ions) de la solution atteint une taille critique. La nucléation hétérogène, induite par des particules externes (poussières de quelques microns) demande une énergie moindre et donc se passe à plus basse sursaturation : il s'agit de nucléation hétérogène primaire. A un niveau de sursaturation plus bas encore, se place la nucléation hétérogène secondaire, qui par définition [22] résulte de la présence d'un cristal parent à une sursaturation telle qu'il n'y ait pas de nucléation primaire.

L'observation du processus de nucléation montre qu'il ne se passe qu'au-dessus d'une certaine sursaturation, donc qu'il existe une zone métastable dans laquelle n'a lieu aucune nucléation. La vitesse 
de nucléation peut être obtenue à partir de la détermination de la largeur de cette zone par deux méthodes :

a) On opère à sursaturation croissante et on détermine la sursaturation pour laquelle on détecte les premiers cristaux. En écrivant que pour cette valeur la vitesse de nucléation est égale à la vitesse de variation de la sursaturation, Nyvlt [23] détermine les coefficients de l'équation empirique donnant la vitesse de nucléation

$$
J=k_{\mathrm{N}} \sigma^{m}
$$

avec $\{m$ : coefficient souvent supérieur à 1 ,

$\{$ : sursaturation,

$k_{\mathrm{N}}$ : coefficient cinétique.

Le coefficient $k_{\mathrm{N}}$ dépend de l'intensité de l'agitation. De telles études menées en cristallisation industrielle montrent qu'il faut non seulement caractériser le flux de solution (par exemple, par un nombre de Reynolds) mais aussi l'énergie d'impact sur les particules en suspension ainsi que les collisions cristal-cristal ou cristal-agitateur [24]. Par exemple, Johnson et al. [25] trouvent une nucléation diminuée quand les agitateurs sont faits d'un matériau mou (plastique, polythène) qui absorberait mieux les chocs.

b) On opère à sursaturation constante et on détermine le temps $t_{\mathrm{L}}$ au bout duquel on détecte les premiers cristaux : il s'agit du temps de latence que l'on confond souvent avec le temps d'induction à forte sursaturation. Le temps de latence $t_{\mathrm{L}}$ est alors pris inversement proportionnel à la vitesse de nucléation [26]. Joshi et al. [27] montrent sur ADP, que le temps de latence est réduit de. 1800 à $200 \mathrm{~s}$ quand on fait croître l'agitation de 0 à 500 tours/min, pour une même sursaturation. Mikhnevich et al. [28] étudient l'effet du temps d'agitation sur la nucléation du sucrose en déterminant la distribution de la taille des particules. Pour une vitesse d'agitation (200 tours/min) et une sursaturation donnée, le temps de latence est fixé; le nombre maximal de particules est obtenu pour un temps d'agitation égal au temps de latence $t_{\mathrm{L}}$. Faisant varier la vitesse d'agitation, ces auteurs observent une diminution de la taille des particules pour des agitations croissantes [29].

Pour toutes les mesures de la largeur de la zone métastable, une erreur systématique vient du mode de détection et donc de la sensibilité du détecteur, œil ou compteur électronique. On est en deçà de la zone métastable et le processus de croissance est déjà entamé.

Une nouvelle méthode proposée par Dugua et Simon [30] est basée sur un tout autre principe : ils mesurent l'évolution de la masse $M$ de cristaux obtenus par précipitation dans une solution à sursaturation constante. Cette fonction $M(t)$ dépend non seulement de la vitesse de nucléation mais aussi du volume de la solution et de l'évolution de la masse de chaque cristal ; par déconvolution de cette fonction, ils obtiennent la vitesse de nucléation, en fonction du temps. Ils montrent que la nucléation peut se décomposer en deux étapes : une nucléation primaire de faible vitesse qui démarre après un temps d'induction et une nucléation secondaire beaucoup plus rapide due aux cristaux déjà formés. Opérant dans des solutions fortement agitées (entre 400 et 1200 tours/min), ils obtiennent une vitesse de nucléation augmentée dans ses deux étapes, et démarrant plus tôt. Le coefficient apparent de diffusion augmenterait avec l'état d'agitation de la solution, ce qui réduirait l'énergie d'activation apparente.

Pour comprendre le phénomène de nucléation secondaire, si important en cristallisation industrielle, Belyustin et Rogacheva [31, 32, 33] d'une part, Denk et Botsaris [34], d'autre part, utilisent le fait que des cristaux de sulfate de magnésium $\left(\mathrm{MgSO}_{4}\right.$, $7 \mathrm{H}_{2} \mathrm{O}$ ) et de chlorate de sodium existent sous deux formes énantiomorphes. Si on place dans une solution sursaturée, un germe gauche, on pourra déterminer si l'origine des noyaux est le germe ou la solution. Denk et Botsaris tracent en fonction de la sursaturation le pourcentage de cristaux ayant la même structure que le germe en solution statique ou agitée (Fig. 2). Par rapport à une cristallisation sans germe, la zone métastable est abaissée, ceci d'autant plus que la solution est plus agitée (domaine étudié : 25 à 350 tours/min). Le mécanisme n'est pas unique puisqu'aux faibles sursaturations seulement $60 \%$ des cristaux ont la même structure que le germe, alors que pour des valeurs plus fortes, on n'obtient que des cristaux de même structure que le germe. Botsaris [35] passe en revue les origines possibles : il peut s'agir de dendrites ou de protubérances faiblement fixées au cristal qui, en se détachant, sont emmenées loin du cristal dans des régions de plus forte sursaturation où elles peuvent survivre [36] une autre possibilité est la formation d'embryons dans la couche limite au voisinage du cristal parent, car il existe dans cette zone un gradient d'impuretés ; une troisième possibilité serait que dans cette couche limite, les molécules d'eau auraient un certain ordre qui modifierait la solubilité de la substance. L'agitation a alors pour effet de modifier la couche limite et d'emmener les particules formées dans la solution.

Une autre façon d'étudier l'influence d'un flux de solution est d'examiner la qualité des cristaux formés.

III. Qualité cristalline. - Les méthodes utilisées sont diverses; citons :

- L'observation optique de la forme des cristaux, des inclusions macroscopiques visibles sur le cristal massif. Par des méthodes interférométriques, ces défauts peuvent être corrélés à l'environnement immédiat du cristal.

- La croissance en présence d'impuretés connues 


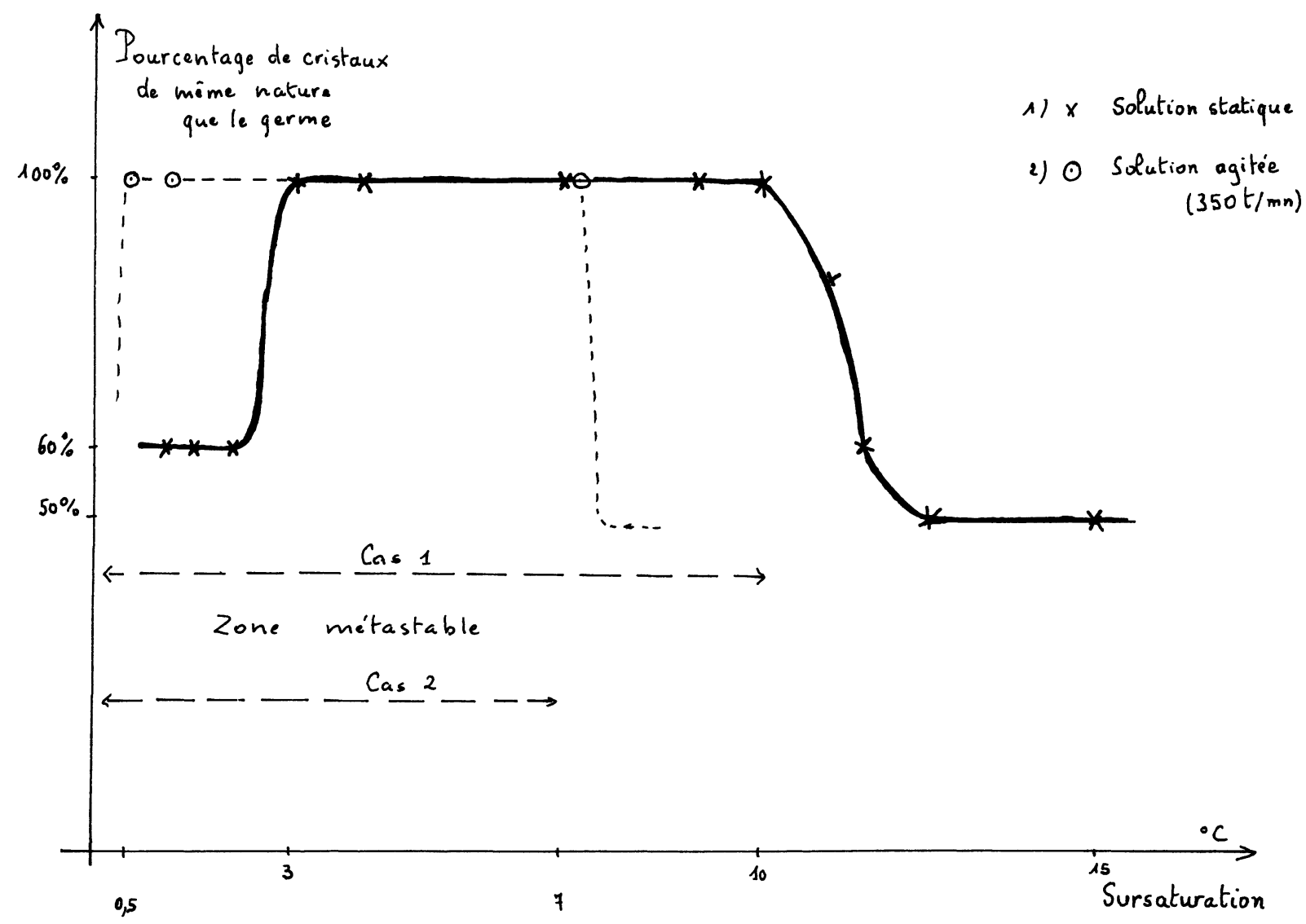

Fig. 2. - Type de cristaux (droits ou gauches) produits en solutions statique et agitée. Cas de $\mathrm{NaClO}_{3}$ [34].

[Type of product crystals obtained in stagnant and stirred solutions. Case of $\mathrm{NaClO}_{3}$ [34].]

qui peuvent même être marquées rendant ainsi possibles des observations radiographiques directes.

- La topographie aux Rayons X qui permet de détecter des défauts cristallins en volume.

Des défauts dépendant fortement de l'agitation sont ainsi mis en évidence : changements de faciès, inclusions, dislocations.

A. - Changement de faciès. - Plusieurs auteurs $[37,4,38]$ ont montré que l'agitation de la solution conduit pour des cristaux tels que ADP, KDP, à un phénomène de tapering. Pour ces cristaux allongés selon l'axe $c$, deux faces prismatiques opposées normalement parallèles forment, en solution agitée, un angle dont la valeur dépend de la vitesse de rotation : ainsi dans le cas d'ADP [4] cet angle atteint $10^{\circ}$ pour des vitesses de 3000 tours $/ \mathrm{min}$.

B. - InClusions. - Des inclusions se forment sur des cristaux même en solution non agitée [17]. Bunn [39] et Berg [40] ont montré par des méthodes optiques que la sursaturation varie le long d'une face; elle est plus forte aux arêtes et aux sommets : il s'établit alors localement des conditions de soussaturation qui aboutissent à des inclusions de liqueur mère.

Une faible agitation due à la convection naturelle peut conduire à la formation d'inclusions suivant la position des faces dans le cristallisoir. Goldsztaub et Itti [1] trouvent des inclusions sur trois des six faces tétraédriques d'un alun d'ammonium : au voisinage des facettes dirigées vers le haut, des courants de convection peuvent s'établir, ce qui élève la concentration au voisinage de ces faces conduisant à une croissance dendritique donc à des inclusions. Ce deuxième mécanisme avait déjà été mentionné par Buckley [41]. Un même phénomène se produit à la fin de la croissance d'un gros monocristal dans un récipient de petite taille : ce cristal empêche par son volume un mélange correct de la solution; ainsi une partie de la solution plus concentrée donc plus lourde se trouve confinée en bas du cristallisoir, de telle sorte que les faces supérieures mal alimentées présentent des inclusions [42].

Brooks et al. [43] étudient la formation d'inclusions dans des solutions bien agitées sur toute une série de monocristaux. Certaines inclusions sont reliées à l'histoire du cristal, notamment à des changements de sursaturation pouvant provoquer l'apparition de faces rapides qui, en disparaissant, développent des inclusions.

Abordons maintenant plus en détail quelques études systématiques : Jannsen Van Rosmalen et al. [44, 45] ont essayé de relier la formation 
d'inclusions aux turbulences créées dans le flux de solution. Les cristaux germes (Fig. 3) sont montés sur un agitateur tournant de telle sorte que le flux reçu par les cristaux est parallèle à l'axe $c$. Pour de

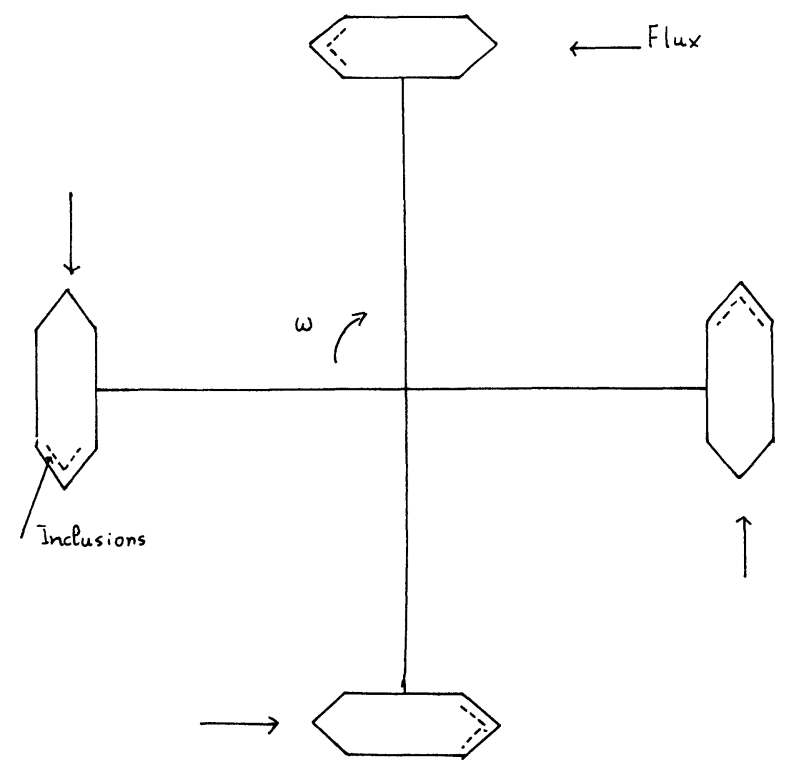

Fig. 3. - Etudes de croissance de KDP pour divers flux [45].

[Growth systems for KDP crystals growing under different fluxes [45].] faibles vitesses du flux, des alignements d'inclusions apparaissent en fin de croissance, dans les faces pyramidales, mais seulement à l'arrière du flux. De plus, ces inclusions ne se développent qu'à une certaine distance de l'arête entre faces prismatique et pyramidale. Pour des flux élevés (c.-à-d. pour des nombres de Reynolds élevés), les inclusions disparaissent. De telles observations ont déjà été faites par Carlson [13] : assimilant le flux de solution à un flux de chaleur, il calcule quelle distance la solution peut parcourir le long d'une face avant d'être soussaturée, donc de conduire à la formation d'inclusions. Van Rosmalen montre que, contrairement à ce que suppose Carlson, le flux au niveau de ces faces n'est plus laminaire. Pour simuler les perturbations du milieu liquide au voisinage du cristal, elle photographie les turbulences qui apparaissent à l'arrière d'un modèle de même forme que le cristal. Ces expériences sont faites dans un flux d'air dont la vitesse est calculée pour donner le même nombre de Reynolds que celui utilisé lors de la croissance. De la fumée est injectée à l'avant pour visualiser les courants. Dans le cas d'un nombre de Reynolds élevé, le flux se propage le long des faces prismatiques puis décolle de la face pyramidale : le flux est alors tourbillonnaire, mais ces tourbillons sont rapidement emmenés loin de la face en croissance de telle sorte que la solution est bien renouvelée et la croissance est bonne. Pour a)
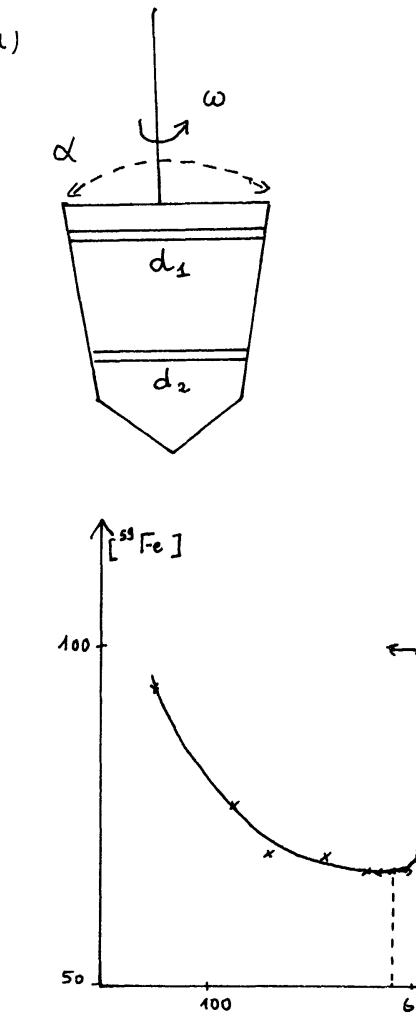

c)

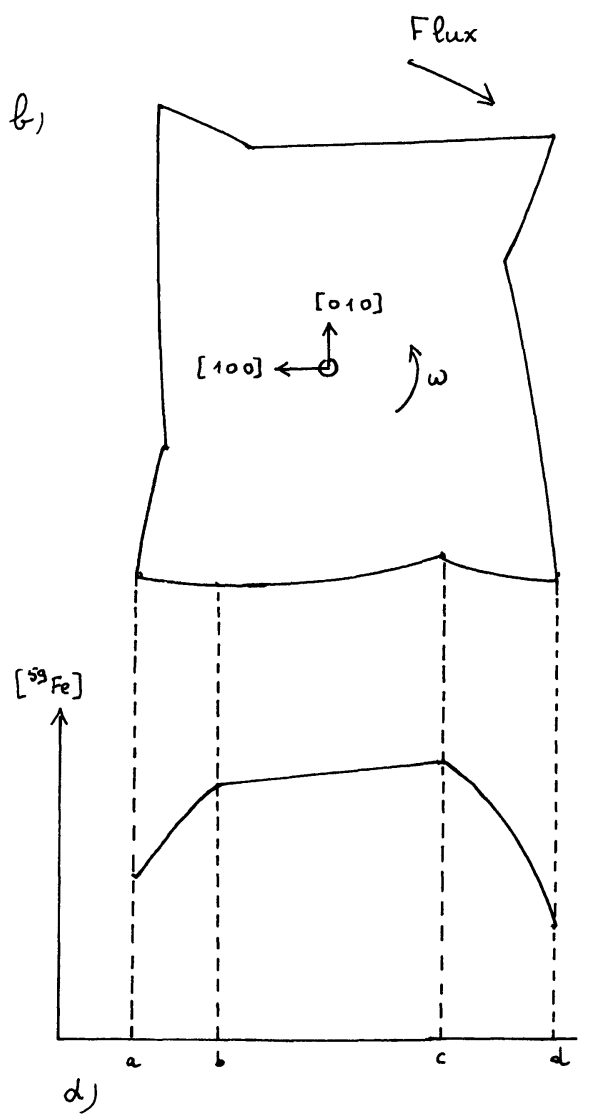

Fig. 4. - Etude de l'incorporation de Fe dans KDP en fonction de l'agitation [46].

[Influence of stirring on Fe incorporation in KDP crystals [46].] 
les flux faibles, les tourbillons qui prennent naissance à une certaine distance de l'arête, restent piégés à l'arrière pendant un temps très long ; ainsi la solution s'appauvrit et des inclusions apparaissent là où il y a un saut de sursaturation, donc à une certaine distance $\mathrm{du}$ bord de la face.

Le KDP a fait l'objet de nombreuses autres études, parmi celles-ci on peut citer les travaux de Belouet [46] qui établissent une relation entre l'incorporation d'impuretés (comme le fer ou le calcium) et l'état d'agitation de la solution. Belouet travaille dans des conditions complètement différentes de Van Rosmalen : sursaturation plus faible, orientation du germe différente. Celui-ci tourne (Fig. 4a) autour de l'axe $c$, le flux est alors reçu sur les faces prismatiques et non sur les faces pyramidales. Pour étudier l'incorporation en $\mathrm{Fe}$, la croissance a lieu en présence de $\mathrm{Fe}$ marqué. Pour des vitesses de rotation élevées, les faces prismatiques ne sont plus planes mais présentent un faciès en corne (Fig. $4 b$ ) ; à partir de ce faciès, il est possible de retrouver des faces prismatiques planes en arrêtant l'agitation ou en jouant sur le taux de fer. En effet, une autoradiographie montre que le fer s'incorpore surtout dans les secteurs prismatiques; une analyse le long d'un front de croissance prismatique courbe montre que la teneur en Fe varie le long d'une telle face : elle chute brutalement de $c$ à $d$ (Fig. 4c) soit donc dans la partie de la face non directement exposée au flux de solution. De plus, dans les secteurs prismatiques, l'incorporation du fer est d'autant plus forte que l'agitation diminue. De façon à relier cette incorporation à une caractéristique du flux, Belouet utilise le fait que deux faces prismatiques poussent en faisant un certain angle $\alpha$ : il obtient ainsi pour un même état d'agitation des lames de dimensions différentes sur lesquelles on peut suivre l'incorporation en $\mathrm{Fe}$ pour une même bande de croissance. Les résultats concernant deux vitesses de rotation sont reportés figure $4 d$. La concentration en $\mathrm{Fe}$ varie en fonction d'un paramètre $\Gamma=\omega d^{2}$, qui est à un facteur près le nombre de Reynolds. Il détermine ainsi un nombre de Reynolds critique pour lequel le taux d'incorporation en $\mathrm{Fe}$ est minimal.

La figure 5 présente une autoradiographie d'une lame issue d'un cristal de KDP obtenu en présence de calcium marqué. Le calcium, contrairement au fer, ne s'incorpore pas au réseau, les faces prismatiques demeurent planes quelle que soit la vitesse de rotation. Mais on remarque sur les portions de faces abritées, des micro-inclusions (l) qui diminuent de taille vers le centre. Des macro-inclusions ont déjà été observées sur des parties de faces abritées quand l'agitation est faite dans un seul sens (2). Ces inclusions peuvent, comme nous l'avons déjà montré, être reliées à des zones de turbulence à l'arrière du cristal. Une autre interprétation qui tient compte des modifications du cristal, est proposée par Belouet : il montre, en effet, que l'incorporation du fer est plus importante à l'arrière du flux; elle crée donc en cet endroit un état de contrainte qui empêcherait une nucléation correcte et aboutirait à la formation de ces microinclusions observées (Fig. 5).

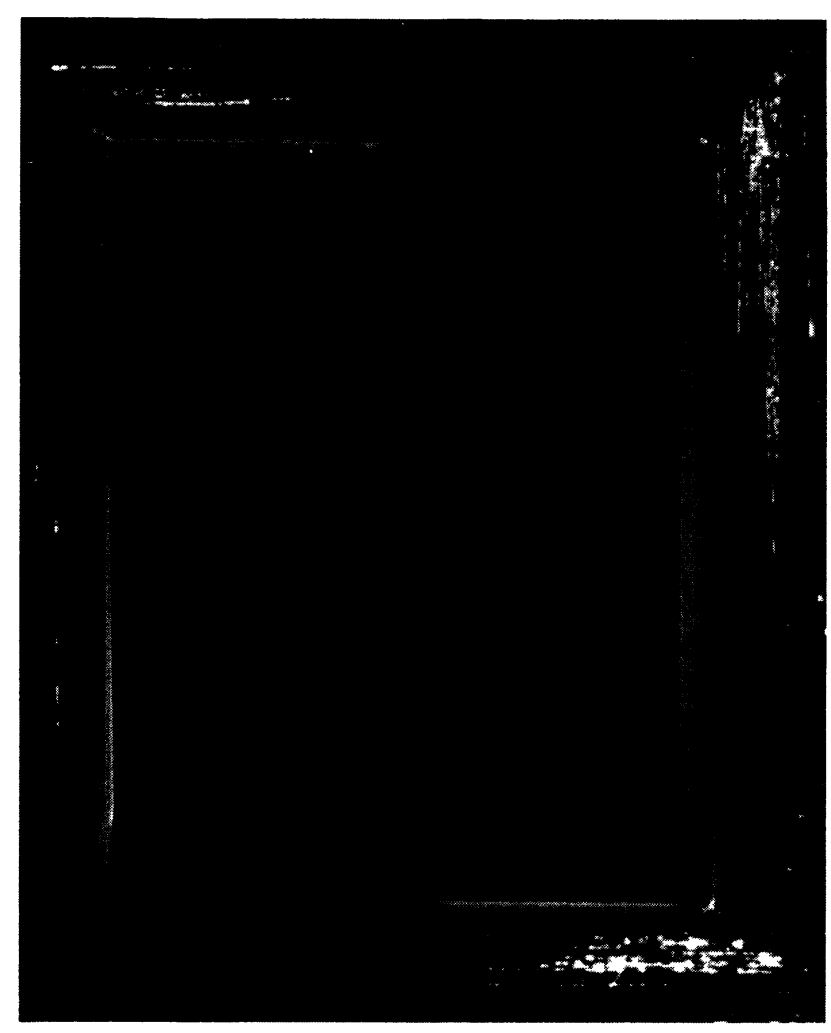

Fig. 5. - Autoradiographie d'une lame (001) de KDP dopé au calcium $(\times 10)$ (publiée avec l'aimable autorisation de C. Belouet).

[Autoradiograph of a (001) slice of KDP doped with marked Ca $(\times 10)$. (Courtesy of C. Belouet).]

C. - Dislocations. - Une mise en évidence plus complète des défauts provoqués par le flux peut être faite par topographies aux rayons $\mathrm{X}$ selon la méthode de Lang. Nous avons entrepris une étude systématique [47] de l'influence du flux sur la croissance d'un cristal (l'alun de potassium) obtenu par une méthode de descente en température. Nous présentons ici une étude faite sur germe (1ī0) [48]. Les paramètres que nous avons fait varier sont sursaturation et vitesse du flux (Fig. 6). Une lame (110) issue du cristal I présente en topographie (Fig. 7) les caractéristiques suivantes : face au flux, de nombreuses dislocations issues du germe se propagent dans le secteur du dodécaèdre puis sont réfractées vers les secteurs octaédriques. Certaines de ces dislocations ont une forte composante vis, ce qui entraîne la disparition de la face en D ; à l'abri du flux, des inclusions liquides de grande taille font que les secteurs octaédriques sont bien meilleurs. Ainsi la présence d'un flux de solution peut entraîner une répartition asymétrique de défauts. Une distribution symétrique est obtenue, à même sursaturation, soit en augmentant le flux (Fig. 8 : alors des dislocations plus nombreuses sont 


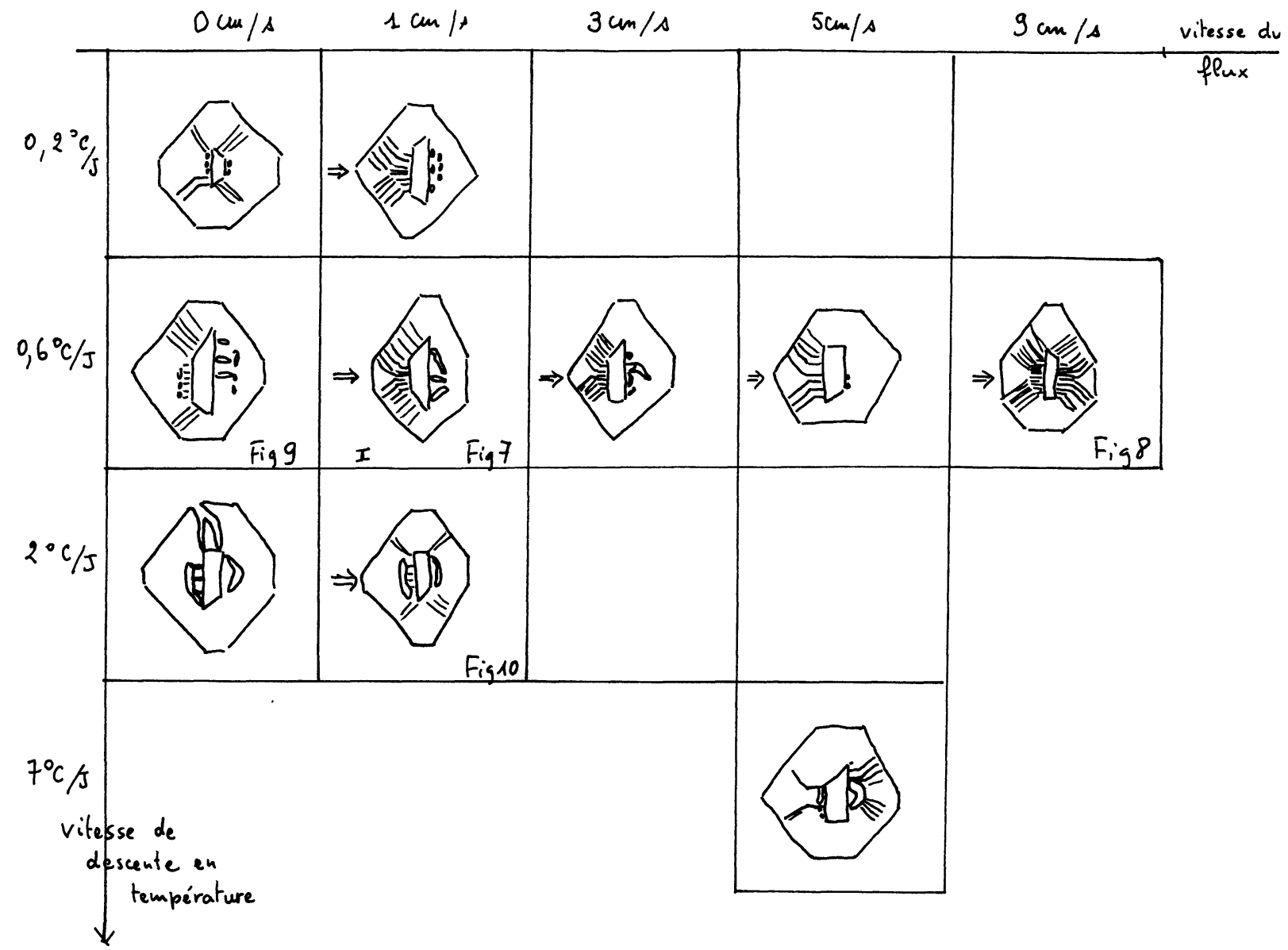

Fig. 6. - Défauts observés sur des germes (110) d'alun de potassium poussés dans diverses conditions de sursaturation et de flux. [Scheme of defects observed in crystals grown from (110) seeds given for various sursaturations and flux velocities.]

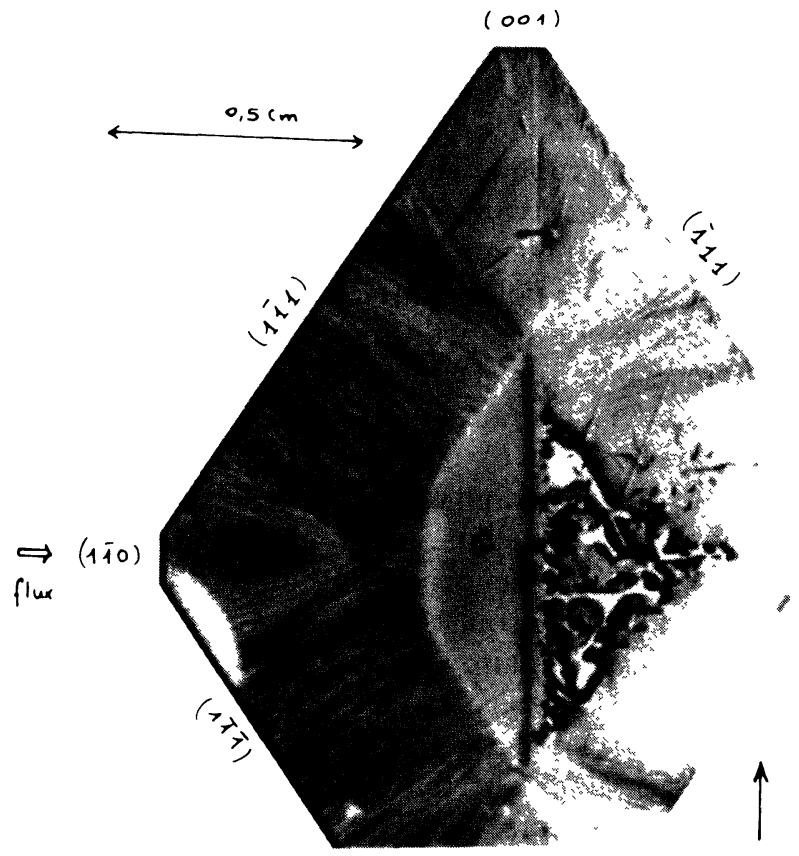

Fig. 7. - Topographie d'une lame (110) d'alun de potassium $-\mathrm{CuK} \alpha_{1}$, réf. 220 .

[Topograph of a (110) slice of potash alum - CuK $\alpha_{1}$, Ref. $\left.\overline{2} 20.\right]$

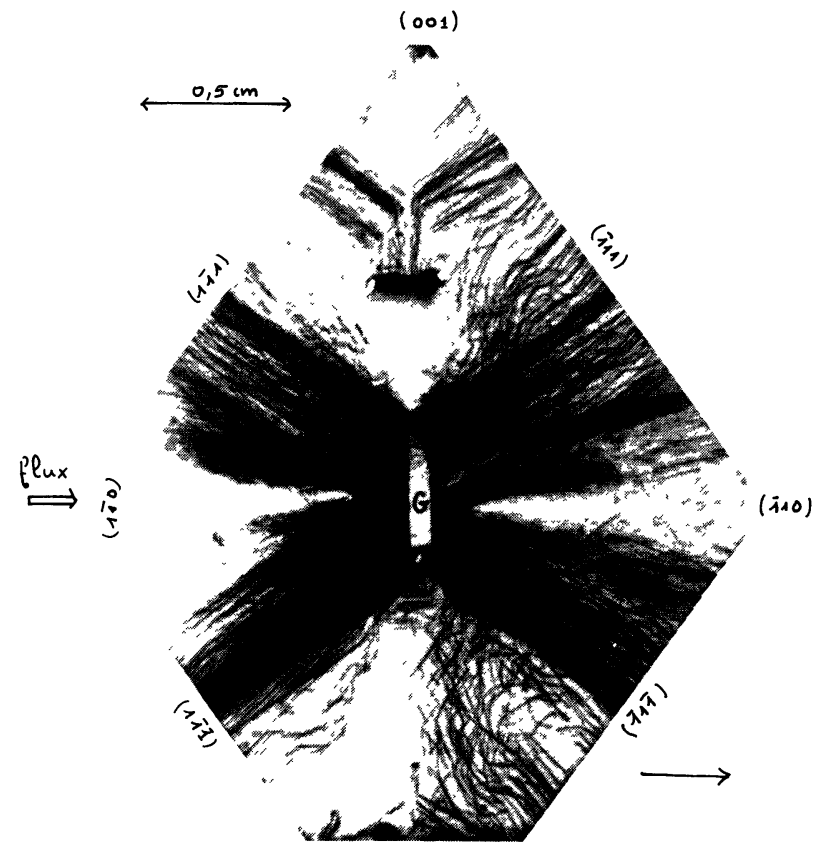

Fig. 8. - Topographie d'une lame (110) d'alun de potassium $-\operatorname{MoK} \alpha_{1}$, réf. 004.

[Topograph of a (110) slice of potash alum $-\operatorname{MoK} \alpha_{1}$, Ref. 004.] 
présentes de part et d'autre du germe), soit en le diminuant (Fig. 9 : il y a alors apparition d'inclusions des deux côtés). En augmentant seulement la sursaturation on retrouve la présence d'inclusions de part et d'autre du germe (Fig. 10). Certaines inclusions parallèles au front de croissance se forment parce qu'à la sursaturation choisie, l'apport de matière, même face au flux est insuffisant : il faut alors beaucoup augmenter le flux pour les voir disparaître. D'autres, plutôt perpendiculaires au front de croissance (Fig. 9) peuvent être dues à une sursaturation trop forte donc à une croissance dendritique de cette face toujours très rapide. Cette double interprétation

\section{(001)}

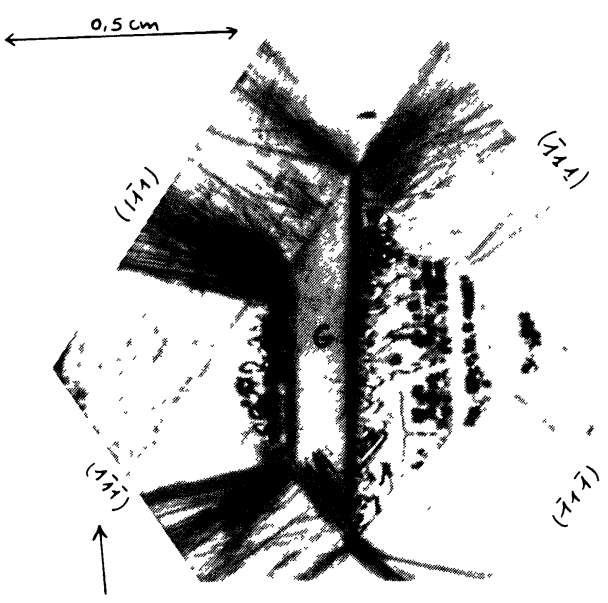

$(\overline{1} 10)$

Fig. 9. - Topographie d'une lame (110) d'alun de potassium $-\operatorname{MoK} \alpha_{1}$, réf. $\overline{2} 20$.

[Topograph of a (110) slice of potash alum - MoK $\alpha_{1}$, Ref. $\overline{2} 20$.]

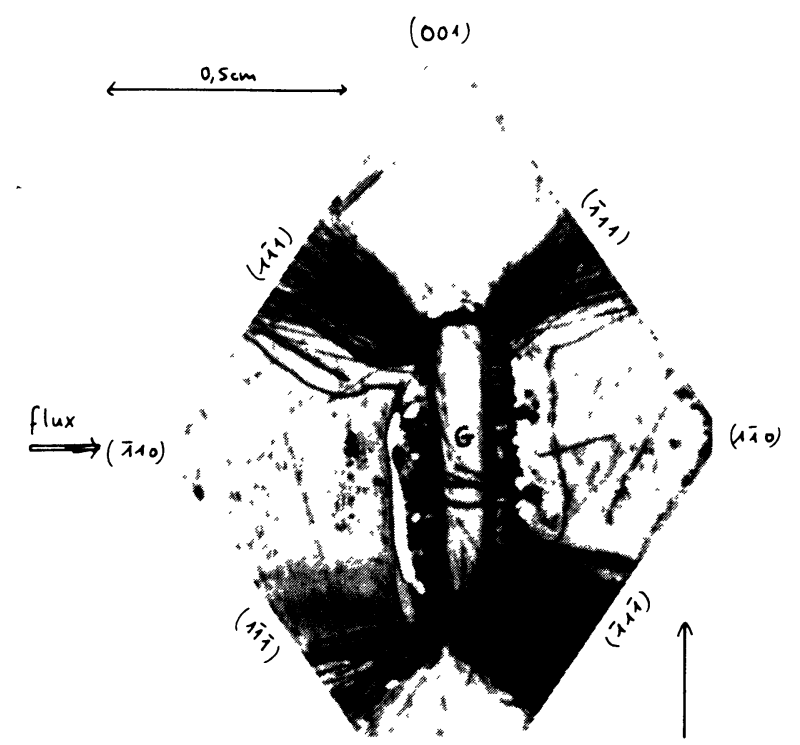

Fig. 10. - Topographie d'une lame (110) d'alun de potassium - $\operatorname{MoK} \alpha_{1}$, réf. 220 .

[Topograph of a (110) slice of potash alum - MoK $\alpha_{1}$, Ref. 220.] est en accord avec les mécanismes précédemment proposés [40, 41]. Quant aux dislocations, Novotny et Moravec [49], trouvent qu'elles sont d'autant plus nombreuses que la sursaturation est plus forte. Kolovskii [50] montre qu'une impureté solide peut provoquer quelquefois la création d'une dislocation vis. Mussard et Goldsztaub [51] constatent, par des méthodes interférométriques combinées à la topographie $\mathrm{X}$, que des dislocations vis peuvent se former au moment et à l'endroit où se produit une variation brusque de sursaturation. Or, un flux de solution peut, d'une part, faire varier localement et brusquement la sursaturation, d'autre part, plaquer sur la face exposée des particules qui sont sources de dislocations le plus souvent à composante coin : en effet, ces particules peuvent être des nucléations parasites, leur fixation incohérente peut conduire à une simple désorientation dans le plan de la face donc à une dislocation coin; or, Denk et Botsaris [8] ont bien montré que le nombre de nucléations parasites augmente avec le flux et la sursaturation.

Ceci peut être illustré dans les deux exemples suivants : pour un cristal tournant sur luimême (Fig. 11), une même face $\mathrm{AB}$ du germe contient les deux types de défauts, la partie droite présente des dislocations donc est face au flux alors que la partie gauche, avec ses inclusions est abritée, ce qui est en accord avec le sens de rotation du cristal. Dans le

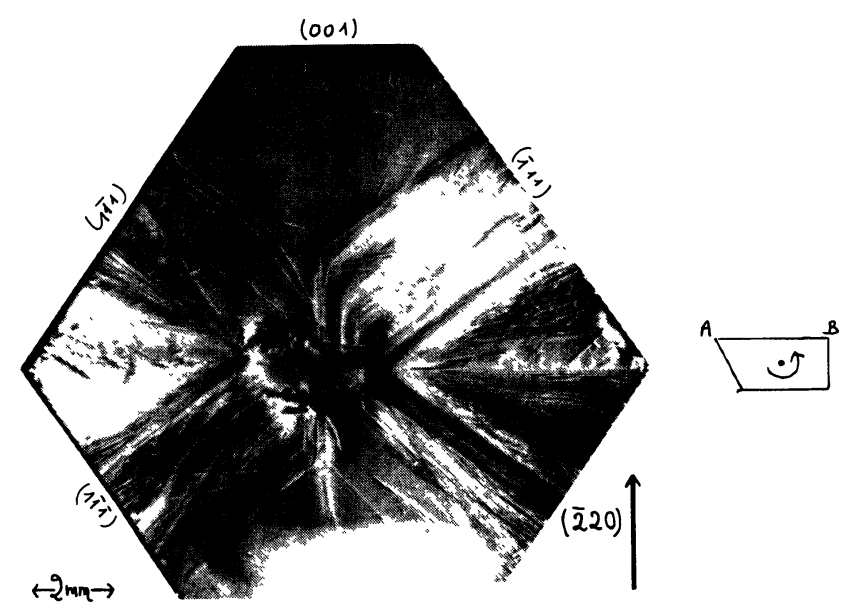

Fig. 11. - Topographie d'une lame (110) d'alun de potassium $-\mathrm{CuK} \alpha_{1}$, réf. 220 .

[Topograph of a (110) slice of potash alum $-\mathrm{CuK} \alpha_{1}$, Ref. 220.]

cas d'une rotation alternée avec changement brusque, on retrouve dislocations et inclusions sur les deux faces (Fig. 12). Au total, l'influence du flux est plus ou moins marquée suivant la valeur qu'on donne aux autres paramètres de croissance mais si on observe une distribution asymétrique de défauts, celle-ci peut être créée par l'existence d'un flux orienté. Cette remarque nous a permis de préciser la direction du flux de solution reçu par un cristal 


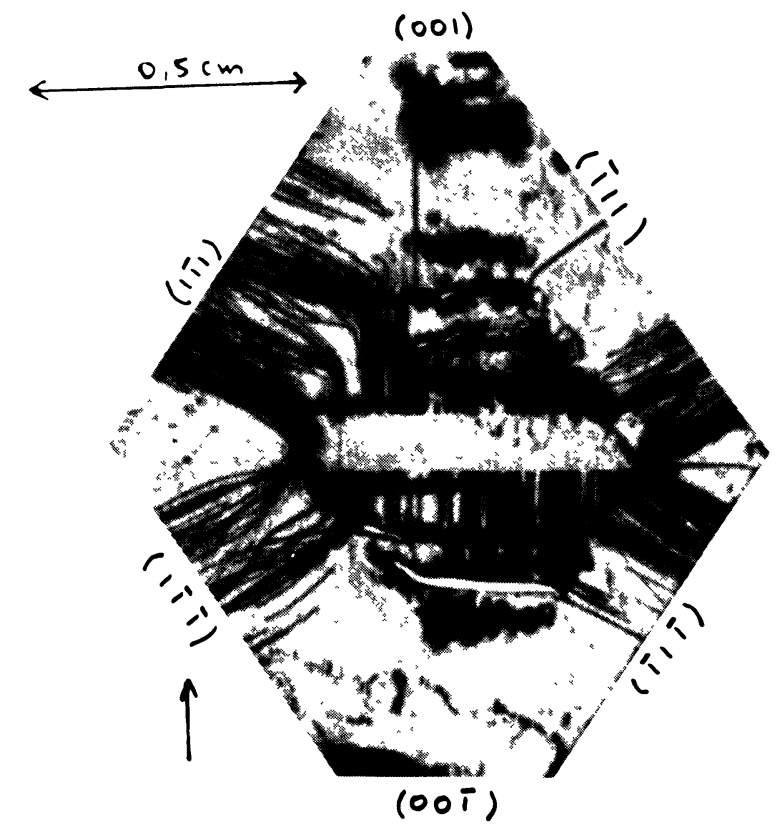

Fig. 12. - Topographie d'une lame (110) d'alun de potassium ayant subi des changements de sens du flux $-\operatorname{MoK} \alpha_{1}$, réf. $\overline{2} 20$.

[Topograph of a (110) slice of potash alum grown with inversions of the direction of the flux $-\operatorname{MoK} \alpha_{1}$, Ref. $\overline{2} 20$.]

de calcite dans un autoclave [52], ou par un cristal de germanate de cobalt et sodium $\left(\mathrm{Na}_{2} \mathrm{CoGeO}_{4}\right)$ [53] poussé, lui aussi, par synthèse hydrothermale. Des cristaux de béryl naturel $[54,55]$ examinés en topographie présentent plusieurs étapes de croissance séparées par des étapes de dissolution : on détecte à la reprise de croissance d'un côté des dislocations, de l'autre des inclusions, ce qui laisse supposer l'existence d'un flux orienté. L'observation des défauts de croissance apporte donc des informations sur les courants de solution auxquels sont soumis les minéraux; de telles informations sont utilisables en géologie pour une meilleure connaissance des gîtes.

IV. Conclusion. - Une revue de la littérature concernant la croissance cristalline permet de constater que chacun des aspects, nucléation, morphologie, nature et distribution des défauts, vitesse de croissance et dissolution, sont affectés par le régime des courants de solution au voisinage du cristal. Ce régime étant modifié du fait même de la croissance, on ne peut préconiser de système unique d'agitation mais on peut comprendre comment réduire ou amplifier certains effets. Cependant les études hydrodynamiques sont insuffisantes puisqu'elles ne tiennent pas compte de la structure des milieux solide et liquide à l'interface. Or la structure atomique détaillée des solutions n'est pas très bien connue et il serait nécessaire d'acquérir des informations sur le type d'ordre qui peut exister dans la solution et, en particulier, au niveau de la couche limite.

\section{Bibliographie}

[1] Goldsztaub, S., Itti, R., C. R. Hebd. Séan. Acad. Sci. 262 (1966) série B 1291-1293.

[2] Petrov, T. G., Treivus, E. G., Kasatkin, A. P., Growing crystals in solution (Cons. Bureau N.Y.) 1969, 61-62.

[3] GiLman, J. J., The art and science of growing crystals (J. Wiley, N.Y.) 1963.

[4] BytevA, I. M., Growth of crystals, vol. 3 (Cons. Bureau N.Y.) 1962, 213.

[5] SiP, V., VANICEK, V., Growth of crystals, vol. 3 (Cons. Bureau N.Y.) $1962,191$.

[6] Bennema, P., Ph. D. Thesis, University of Delft (1965).

[7] Botsaris, G. D., Denk, E. G., Ind. Eng. Chem. Fundam. 9 No 2 (1970) 276-283.

[8] Denk, E. G., Botsaris, G. D., J. Cryst. Growth 6 (1970) 241244.

[9] Mullin, J. W., Garside, J., Trans. Inst. Chem. Engrs 45 (1967) 285-295.

[10] Garside, J., Janssen van Rosmalen, R., Bennema, P., $J$. Cryst. Growth 29 (1975) 353-366.

[11] Scheel, H. J., Elwell, D., J. Electrochem. Soc., Solid State Science and technology 120 No 6 (1973) 818-824.

[12] Levich, V. G., Physicochemical hydrodynamics (Fizmatgiz) 1959.

[13] Carlson, A. E., Growth and perfection of crystals, R. H. Doremus, B. W. Roberts, D. Turnbull (Wiley N.Y.) $1958,421$.

[14] Bennema, P., Crystal Growth, H. S. Peiser (Pergamon Press Oxford) $1967,413$.
[15] Punin, Yu. O., Petrov, T. G., Growth of crystals, vol. 9 (Cons. Bureau N.Y.) 1975, 83-86.

[16] Belyustin, A. V., Portnov, V. N., Sov. Physics Crystallography 7 № 2 (1962) 214-216.

[17] Petrov, T. G., Growth of crystals, vol. 3 (Cons. Bureau (1962) N.Y.) 107.

[18] Shabalin, K. N., Inyushkin, G. V., Growth of crystals, vol. 6A (Cons. Bureau (1968) N.Y.) 13.

[19] Rodrigues-Clemente, R., Acta Geologica Hispanica 11 (1976) 93-95.

[20] Powers, H. E. C., Nature 178 (1956) 139.

[21] GyulaI, Z., Growth of crystals, vol. 3 (Cons. Bureau N.Y.) 1962, 71.

[22] Asselbergs, C. J., De Jong, E. J., Secondary nucleation. Report of the Working Party on Crystallization of the European Federation of Chemical Engineering, Delft 1972.

[23] NyvLt, J., Industrial Crystallization, the present state of the art (Verlag Chemie) 1978.

[24] Clontz, N. A., Mac Cabe, W. L., Chem. Eng. Progr. Symp. Ser. 67 (1971) 6.

[25] Johnson, R. T., Rousseau, R. W., Mac Cabe, W. L., A.I. Ch. E. Symp. Ser. 68 (1972) 31-34.

[26] Van Hook, A., Bruno, A. J., Disc Faraday Soc. 5 (1949) 112.

[27] Joshi, M. S., Antony, A. V., J. Cryst. Growth 46 (1979) 7-9.

[28] Mikhnevich, G. L., Gudz, P. N., Growth of crystals, vol. 3 (Cons. Bureau N.Y.) 1962, 94.

[29] Mikhnevich, G. L., Yanchuk, R. A., Growth of crystals, vol. 3 (Cons. Bureau N.Y.) 1962, 90. 
[30] Dugua, J., Simon, B., J. Cryst. Growth 44 (1978) 265-279.

[31] Belyustin, A. V., Rogacheva, E. D., Growth of crystals, vol. 4 (Cons. Bureau N.Y.) 1966, 3.

[32] Rogacheva, E. D., Belyustin, A. V., Growth of crystals, vol. 5B (Cons. Bureau N.Y.) 1968, 38.

[33] Rogacheva, E. D., Growth of crystals, vol. 7 (Cons. Bureau N.Y.) $1969,173$.

[34] Denk, E. G., Botsaris, G. D., J. Cryst. Growth 13/14 (1972) 493-499.

[35] Botsaris, G. D., Industrial Crystallization, J. W. Mullin (Plenum Press) 1976, 3-22.

[36] Garabedian, H., Strickland-Constable, R. T., J. Cryst. Growth 13/14 (1972) 506-509.

[37] Egli, P. H., Johnson, L. R., The art and science of growing crystals, Gilman (1963) (Wiley N.Y.) 194.

[38] Belouet, C., Acta Electronica 16 (1973) 340-353.

[39] Bunn, C. W., Disc Faraday Soc. 5 (1949) 132.

[40] Berg, W. F., Proc. R. Soc. (London), A 164 (1938) 79.

[41] Buckley, H. E., Crystal Growth (Wiley N.Y.) 1952. 433.

[42] Ribet, J. L., Thèse de Docteur Ingénieur, Montpellier (1972).

[43] Brooks, R., Horton, A. T., Torgesen, J. L., J. Cryst. Growth 2 (1968) 279-283.

[44] Janssen van Rosmalen, R., Bennema, P., J. Cryst. Growth 42 (1977) 224-227.
[45] Janssen van Rosmalen, R., Van Der Linden, W. H., DobBINGA, E., VISSER, D., Kristall und Technik 13 (1978) 17-28.

[46] Belouet, C., Monnier, M., Verplanke, J. C., J. Cryst. Growth 29 (1975) 109-120.

[47] Gits-Leon, S., LefaucheuX, F., Robert, M. C., J. Cryst. Growth 44 (1978) 345-355.

[48] Gits, S., Thèse de III e Cycle, Paris (1978).

[49] Moravec, F., Novotny, J., Kristall und Technik 7-8 (1971) 891-902.

[50] KozlovskiI, M. I., Growth of crystals, vol. 3 (Cons. Bureau N.Y.) $1962,101$.

[51] Mussard, F., Goldsztaub, S., J. Cryst. Growth 13/14 (1972) 445-448.

[52] Lefaucheux, F., Robert, M. C., Authier, A., J. Cryst. Growth 19 (1973) 329-337.

[53] Demianiets, L. N., Duderov, N. G., Lobachev, A. N., Lefaucheux, F., Robert, M. C., Authier, A., J. Cryst. Growth 44 (1978) 570-580.

[54] Gits-Leon, S., Robert, M. C., Zarka, A., Bull. Minéral. 101 (1978) 399-402.

[55] 'SCandale, E., Scordari, F., Zarka, A., J. appl. cryst. 12 (1979) 78-83. 